\title{
EVOLUCIÓN POBLACIONAL Y PARÁMETROS REPRODUCTIVOS DEL AGUILUCHO CENIZO EN EL INTERIOR DE LA PROVINCIA DE CASTELLÓN (NE ESPANAA)
}

\section{R. Limiñana \& V. Urios}

EsTACIÓN BIOLÓGICA TERRA NATURA

CibiO, Universidad de Alicante - Fundación Terra Natura

ruben.lm@ua.es

En el año 2003, el CIBIO con la colaboración y el apoyo de la Conselleria de Territori i Habitatge de la Generalitat Valenciana, inicia un estudio con el fin de conocer mejor las poblaciones de aguilucho cenizo (Circus pygargus L.) en la Comunidad Valenciana, haciendo especial hincapié en la población del interior de Castellón, motivado por la inminente pérdida de hábitat que supone la instalación del Aeropuerto de Castellón en una de las principales zonas ocupadas por el aguilucho cenizo en esta provincia. Esta población del interior de Castellón es una de las pocas poblaciones españolas de esta especie que usa todavía un hábitat natural como sustrato para la nidificación, además de ser una de las escasas poblaciones europeas que están en expansión y aumento del tamaño poblacional desde que empezaron a colonizar este tipo de sustratos a principios de los años 80 . Por lo tanto, el estudio de esta población parece, en principio, muy interesante para la conservación de la especie incluso a mayor escala.

El objetivo del presente proyecto es conocer determinados aspectos de la biología de esta especie que sirvan como punto de partida para proponer medidas adecuadas para su conservación en la Comunidad Valenciana. Para ello se pretende realizar una serie de trabajos que ayuden a este objetivo, como son los que se indican a continuación:

Localización y seguimiento de nidos: durante cada temporada de nidificación se trata de localizar el mayor número de nidos posible de aguilucho cenizo, visitando zonas ocupadas por esta especie en años anteriores así como prospectando nuevas zonas que en principio tienen condiciones adecuadas para ser ocupadas por esta especie. Una vez localizados los nidos se procede a realizar un seguimiento de los mismos con el fin de poder calcular los parámetros reproductivos de las diversas pobla- 
ciones (tamaño de puesta, tamaño de pollada, productividad, porcentaje de nidos con éxito), así como tomar medidas biométricas de los pollos para calcular tasas de crecimiento de los mismos (Figura 1).

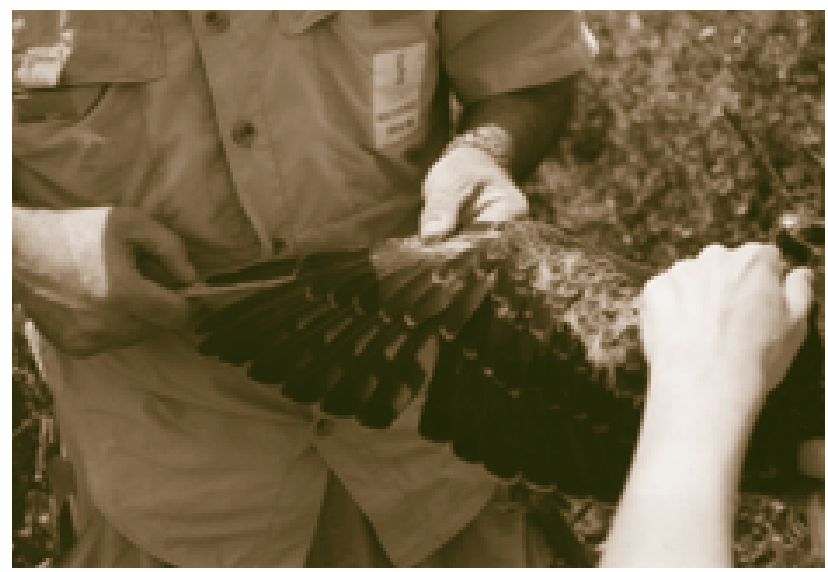

Figura 1. Toma de medidas biométricas en pollos de aguilucho cenizo en edad de vuelo.

Durante las visitas a los nidos se procederá a anillar los pollos con anillas de PVC de lectura a distancia (Figura 2), siempre que se encuentren en la edad adecuada. Esto servirá para mantener indivi-

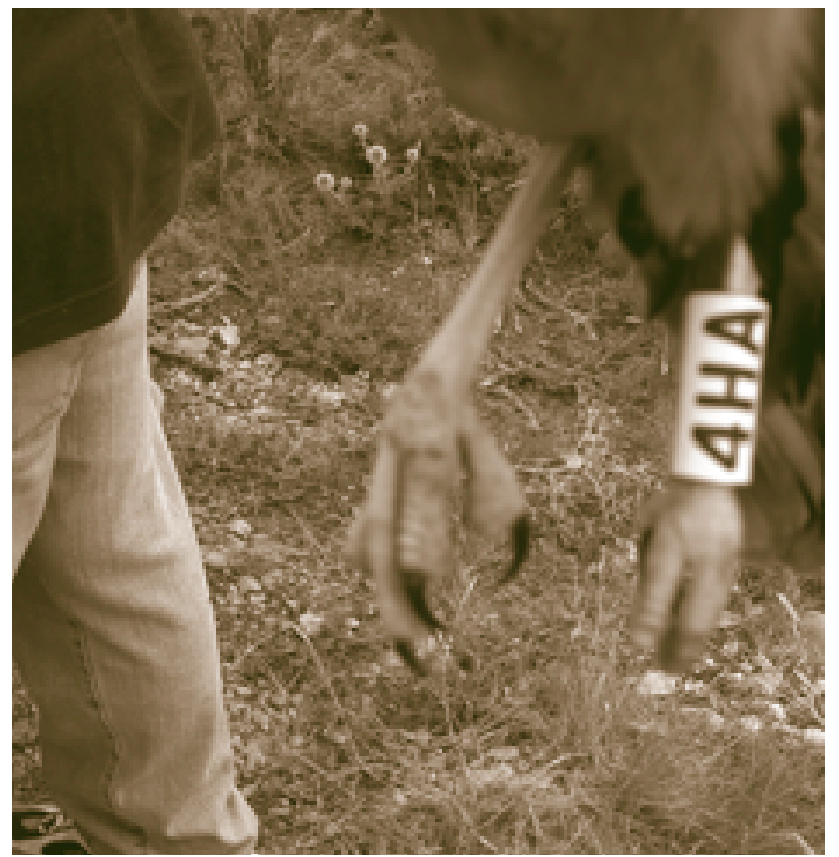

Figura 2. Anilla de PVC con inscripción alfanumérica; con este tipo de marcas se consigue mantener individualizados a los pollos nacidos en la zona con el fin de poder obtener recuperaciones en años posteriores. dualizados a los distintos ejemplares, así como para ver si los aguiluchos regresan a criar a las zonas donde nacieron o si existe una alta dispersión juvenil.

En estas mismas visitas se intentará obtener muestras de sangre de los pollos para realizar estudios de hematología y bioquímica que permitan establecer la condición fisiológica de los pollos, así como poder detectar algún tipo de contaminantes, como pueden ser los biocidas.

- Estudios de alimentación de las distintas poblaciones de la especie, basados en el análisis de egagrópilas y restos de presas encontrados en los nidos o en posaderos de esta especie, así como mediante observaciones directas en el campo.

- Caracterización del hábitat de nidificación del aguilucho cenizo. Esto es especialmente importante en el caso de la población del interior de Castellón, la cual se encuentra sometida a una inminente pérdida de hábitat por la construcción del futuro aeropuerto de Castellón.

- Radioseguimiento de ejemplares adultos de aguilucho cenizo. Los ejemplares serán marcados con emisores de telemetría convencional de tipo mochila. Con este seguimiento se pretende conocer los territorios vitales de la especie, así como conocer las zonas a donde esta especie se desplaza a cazar.

\section{SITUACIÓN ACTUAL DE LAS POBLA- CIONES DE AGUILUCHO CENIZO EN LA COMUNIDAD VALENCIANA}

La nidificación de esta especie en la Comunidad Valenciana se conoce desde antiguo. Hasta principios de la década de los 80 el aguilucho cenizo únicamente nidificaba en humedales, presentando dos núcleos poblacionales, uno en el Prat de Cabanes Torreblanca, en el litoral de Castellón y otro en las Lagunas de La Mata y Torrevieja, en el sur de Alicante (Ferrer et al., 1986). En ese momento se localizó por primera vez a la especie nidificando en maquias termófilas del interior de Castellón. Desde entonces se inició un programa de seguimiento y protección de la especie en esta provincia, lo que unido a la declaración de la zona litoral como Par- 
que Natural permitió una rápida recuperación de la especie en la provincia (Jiménez y Surroca, 1995). Desde ese momento y hasta la actualidad, Castellón es una de las pocas provincias españolas en las que la tendencia poblacional del aguilucho cenizo es al alza (Arroyo y Pinilla, 1996; García y Arroyo, 2003).

En Alicante, el aguilucho cenizo nidifica en los tres Parques Naturales del sur de la provincia (El Hondo, Salinas de Santa Pola y Lagunas de La Mata-Torrevieja), usando como hábitat de nidificación los saladares - carrizales. La mayoría de las parejas que podemos encontrar en estas zonas húmedas se localizan en el Parque Natural de las Lagunas de La Mata - Torrevieja, donde se conoce la nidificación de esta especie desde antes de los años 70 (Reig, 2002). Esta población sudalicantina ha sufrido grandes oscilaciones (Sánchez et al., 1995). A mediados de los años 70 podíamos encontrar unas 11 parejas, todas ellas localizadas en los saladares de la Salina de Torrevieja y la Laguna de La Mata. La población fue disminuyendo progresivamente hasta contar con sólo 2 parejas en los años 1983 y 1984 . Desde entonces se produjo una recuperación en la población y a finales de los 80 empezó a nidificar en El Hondo, alcanzando de nuevo las 11 parejas en 1992 (Sánchez et al., 1995). Es a mediados de la década de los 90 cuando se localiza como nidificante en las Salinas de Santa Pola. También se ha apuntado la posible nidificación en el Clot de Galvany entre 1998 y 1999 (Ramos et al., 2002).

$\mathrm{El}$ interior de la provincia de Alicante, más concretamente en el Valle de los Alhorines (Villena), es el único punto de la Comunidad Valenciana donde se ha localizado a esta especie nidificando en cultivos de cereal, ya que todas las demás parejas usan algún tipo de vegetación natural como sustrato de nidificación. Desde el año 1999 hasta el 2003 podíamos encontrar 1 ó 2 parejas nidificantes en esta zona, pero en 2004 no se ha podido constatar la nidificación.

Los primeros datos de nidificación del aguilucho cenizo en la provincia de Valencia datan de 1994, cuando la especie es localizada como nidificante en pastizales - juncales de la Marjal del Moro (Sagunto). Desde entonces cada año han criado allí entre 1 y 3 parejas, desapareciendo como nidificante en el año 2002.

Castellón es la provincia más importante para el aguilucho cenizo en la Comunidad Valenciana. Esta especie cría en los humedales litorales de esta provincia desde hace mucho tiempo, donde nidifica en pastizales - juncales. A principios de los años 80 es localizada nidificando en maquias mediterráneas xerófilas del interior de la provincia, posiblemente como consecuencia de la expansión del núcleo litoral (Figura 3). En la actualidad encontramos en este núcleo del interior unas $98-100$ parejas nidificantes, mientras que en la zona litoral se localizaron 34 parejas en 2002 y 21 en 2003 . De esta manera, la población del interior de Castellón es una de las piezas claves para la conservación del aguilucho cenizo en la Comunidad Valenciana e incluso a mayor escala. En el sur de la provincia de Tarragona (región del Montsià), se ha localizado por primera vez a esta especie nidificando en matorrales mediterráneos en Cataluña (Pomarol, com. pers.). Todo parece indicar que estos aguiluchos han llegado allí como consecuencia de la expansión que está sufriendo la población del interior de Castellón.

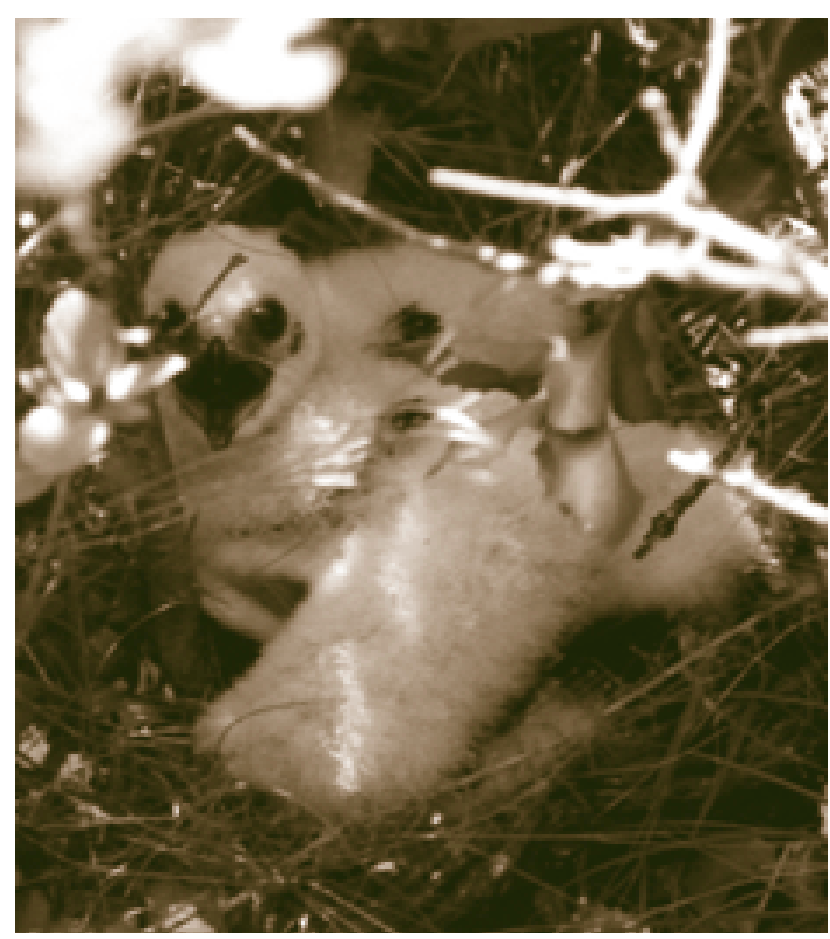

Figura 3. Pollo de aguilucho cenizo de pocos días de edad en un nido ubicado en los matorrales mediterráneos del interior de la provincia de Castellón. 


\section{EVOLUCIÓN DE LA POBLACIÓN NIDI- FICANTE DE AGUILUCHO CENIZO EN EL INTERIOR DE LA PROVINCIA DE CASTELLÓN (1981 - 2004)}

La población de aguilucho cenizo que nidifica en matorrales del interior de Castellón ha experimentado una notable expansión desde principios de los años 80 (Jiménez, 1991). A mediados de esta década, esta especie ocupaba sólo cinco cuadrículas del Atlas de las Aves Nidificantes de la Comunidad Valenciana, parte de los cuales correspondían a la población del Prat de Cabanes - Torreblanca. En el año 2000 ya ocupaba 12 de estas cuadriculas. Esta expansión se ha producido hacia el norte, donde el aguilucho ha ocupado zonas donde la vegetación es similar a la existente en el área donde se detectaron por primera vez a principios de los 80 .

Los primeros datos de nidificación del aguilucho cenizo en los matorrales mediterráneos del interior de la provincia de Castellón son recogidos en el año 1978. Sin embargo no es hasta 1981 cuando esta zona es visitada asiduamente por ornitólogos y a partir de esta fecha es cuando se dispone de datos fiables sobre el tamaño de la población reproductora.

Durante los primeros años de presencia de la especie en estos matorrales interiores no son más de tres las parejas nidificantes. Hasta el año 1988 el número máximo de parejas reproductoras que podemos encontrar es siete. El primer salto importante en cuanto a número de parejas nidificantes se produce en 1989, cuando encontramos un mínimo de 20 parejas. Desde esa fecha, la población experimenta un crecimiento continuo, alcanzando un mínimo de 58 parejas reproductoras en 1999. Es entonces cuando se produce un nuevo salto importante, llegando a las 87 parejas al año siguiente. En el año 2001 se estima un mínimo de 98 parejas reproductoras; la población parece haberse estabilizado en torno a este valor, ya que hasta la actualidad no se ha superado este número de parejas nidificantes (Figura 4).

Con los datos obtenidos durante las visitas a los nidos se pueden calcular los parámetros reproductivos que nos ayuden a evaluar el éxito reproductivo

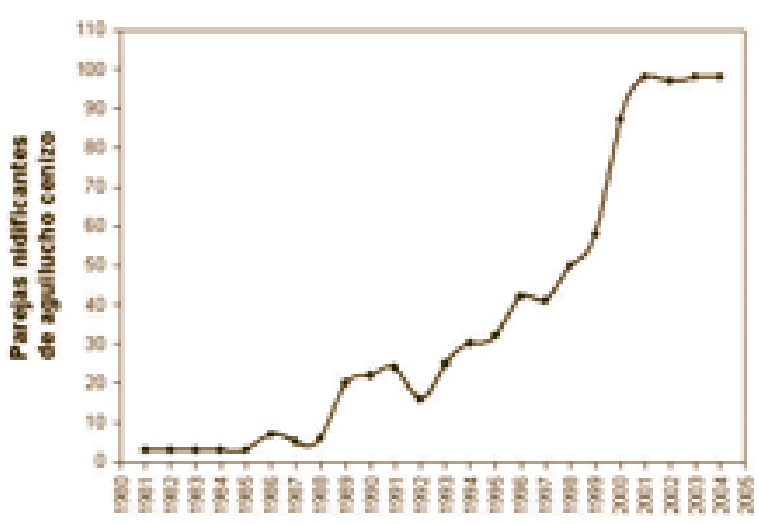

Figura 4. Evolución de la población nidificante de aguilucho cenizo en los matorrales del interior de la provincia de Castellón.

de esta población de aguilucho cenizo. Para ello, se han calculado los siguientes:

- tamaño medio de puesta: número de huevos puestos / $\mathrm{n}^{\mathrm{o}}$ parejas con puesta completa (se han eliminado los nidos depredados, abandonados o expoliados en fase de huevo, ya que la puesta hasta ese momento podría no ser completa)

- tamaño medio de pollada: número de pollos nacidos / número de nidos de los que nace al menos 1 pollo

- productividad: número de pollos que vuelan I número de parejas controladas (entendiendo por pareja controlada aquella de la que se sabe con toda seguridad el número de pollos que sacan adelante, aunque este valor sea cero)

- \% de nidos con éxito: número de nidos en los que vuela por lo menos 1 pollo / número de parejas controladas

- \% vuelo: número de pollos que vuelan / número de pollos nacidos (usando para ello los datos de nidos de los que se conoce con seguridad tanto los pollos nacidos como los que llegan a volar).

El tamaño de puesta para el periodo 1989 - 2004 se ha determinado a partir de los datos obtenidos de 304 nidos. Se ha obtenido un valor medio de $4.29 \pm$ 0.87 huevos / pareja, siendo el tamaño de puesta más frecuente el de 4 huevos ( $\mathrm{n}=133$ nidos; $43.8 \%$ ), seguido de las puestas de $5(\mathrm{n}=112 ; 36.8 \%)$ y $3(\mathrm{n}=$ 
39; $12.8 \%$ ) huevos. El mayor tamaño de puesta observado fue de 8 huevos ( $\mathrm{n}=2 ; 0.7 \%)$.

Para este mismo periodo se ha obtenido un tamaño medio de pollada en eclosión de $3.64 \pm$ 0.98 pollos / pareja $(\mathrm{n}=331$ nidos $)$, con un tamaño modal de 4 pollos en eclosión ( $\mathrm{n}=146$ nidos; $44.1 \%$ ) y una productividad media de $2.75 \pm 1.46$ pollos en vuelo / pareja $(\mathrm{n}=390$ nidos $)$, siendo el valor modal 3 pollos en vuelo $(\mathrm{n}=131 ; 33.6 \%)$.

Por término medio, el $86.57 \%$ de los pollos que nacen llegan a volar $(\mathrm{n}=352$, rango: $72.7-$ $100 \%)$. El porcentaje de nidos con éxito en esta zona varió entre el 65.9 y el $100 \%$, obteniéndose por término medio en el periodo de estudio un $84.36 \%$ de nidos con éxito $(\mathrm{n}=390)$.

Los dos parámetros más importantes que reflejan el verdadero éxito reproductivo en esta especie son el porcentaje de nidos con éxito y la productividad. El porcentaje de nidos con éxito en el interior de Castellón es más alto que el que se ha encontrado en otras poblaciones ibéricas (Arroyo, 1996; Corbacho y Sánchez, 2000) incluso si tenemos en cuenta sólo los nidos en los que se ha producido un manejo para evitar en parte la mortalidad producida por las cosechadoras. La productividad de esta población es también superior a las observadas en otras regiones de Europa (Castaño, 1997; Corbacho et al., 1997; Pandolfi y Giacchini, 1991), lo que viene condicionado en parte por el hecho de que la nidificación se realiza en manchas de vegetación natural, donde la mortalidad es menor que en zonas donde nidifica en cultivos de cereal (Figura 5).

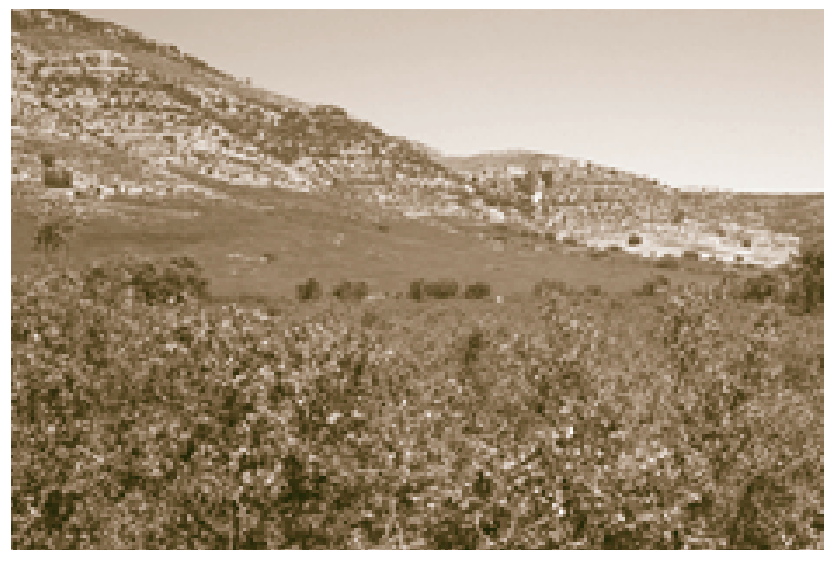

Figura 5. Hábitat de nidificación del aguilucho cenizo en el interior de Castellón.
La conservación de ésta y otras poblaciones que nidifican en hábitat naturales es muy importante, ya que la productividad de estas poblaciones es mayor que la de poblaciones que crían en estepas cerealistas; de esta manera, estas poblaciones pueden actuar como fuentes, proporcionando aguiluchos que podrían reforzar poblaciones más o menos cercanas, a la vez que colonizar nuevas regiones. Sin embargo, es necesario que en otras provincias de España se intente hacer lo posible para que esas poblaciones no sean un sumidero donde se vayan perdiendo gran parte de los aguiluchos que llegan procedentes de estas zonas de vegetación natural.

\section{AGRADECIMIENTOS}

Gran parte de los datos aquí presentados corresponden al trabajo de seguimiento y conservación de la población de aguilucho cenizo en Castellón que viene realizando la Generalitat Valenciana desde 1988, y han sido facilitados por la D.G. de Gestión del Medio Natural (Consellería de Territorio y Vivienda). Muy importante en este sentido es el trabajo realizado por el personal del Centro de Recuperación de Fauna "Forn del Vidre" (Martín Surroca, Stephan Miralles, Sara Ferreras y Pepe Durall).

\section{BIBLIOGRAFÍA}

ARROYO, B. 1996. Reproductive success of Montagu's Harrier (Circus pygargus) and Hen Harrier (C. cyaneus) in an agricultural habitat of central Spain. In: Muntaner J. \& Mayol J. (eds.) Biología y conservación de las rapaces mediterráneas, 1994. Monografías SEO/Birdlife 4: 459-463.

ARROYO, B.E. y PINILLA, J. 1996. A critical review of the population estimates of the Montagu's Harrier (Circus pygargus) in Spain: implications for conservation. In: Fernández Gutiérrez J. \& Sanz Zuasti J. (eds.) Conservation of stepparic birds and their habitats: 91-98. Junta de Castilla y Leon, Valladolid.

CASTAÑO, J.P. 1997. Fenología de puesta y parámetros reproductivos en una población de Aguilucho Cenizo (Circus pygargus) en el Campo de Montiel. Ardeola 44(1): 51-59. 
CORBACHO, C. y SÁNCHEZ, J.M. 2000. Clutch size and egg size in the breeding strategy of Montagu's Harrier Circus pygargus in a Mediterranean area. Bird Study 47: 245-248.

CORBACHO, C., SÁNCHEZ, J.M. y SÁNCHEZ, A. 1997. Breeding biology of Montagu's Harrier Circus pygargus L. in agricultural environments of southwest Spain; comparison with other populations in the western Paleartic. Bird Study 44: 166-175.

FERRER, X., MARTÍNEZ, A. y MUNTANER, J. (eds.) 1986. Esparver cendròs. In: Història Natural dels Països Catalans, Vol. 12: 140-141. Barcelona.

GARCÍA, J.T. y ARROYO, B. 2003. Aguilucho cenizo, Circus pygargus. In: Martí, R. y Del Moral, J.C. (eds.) Atlas de las Aves Reproductoras de España: 178-179. Dirección General de Conservación de la Naturaleza - Sociedad Española de Ornitología, Madrid.

JIMÉNEZ, J. y SURROCA, M. 1995. Evolución poblacional y reproducción del Aguilucho Ceni- zo Circus pygargus en la provincia de Castellón. Alytes VII: 287-296.

PANDOLFI, M. y GIACCHINI, P. 1991. Distribuzione e successo riproduttivo di albanella minore, Circus pygargus, nelle Marche. Riv. Ital. Orn. 61 (1-2): 25-32.

RAMOS, A.J., FIDEL, L. y PAVÓN, M.A. 2002. Las aves del Clot de Galvany. Ajuntament d'Elx, Elche.

REIG, A. 2002. En recuerdo del ornitólogo Alfredo Noval Junquera (1928-2001): su correspondencia ornitológica con el alicantino James MacIvor. In: Las aves en Alicante. Anuario Ornitológico de Alicante 2000. SEO-Alicante, Alicante.

SÁNCHEZ, J.A., SÁNCHEZ, M.A., GARCÍA, F.J., EGUIA, S., CALVO, J.F., CARMINA, D., CABALLERO, J. y ORTUÑO, A. 1995. Distribución, estatus y notas sobre la biología del aguilucho cenizo Circus pygargus en Alicante y Murcia. Alytes VII: 339-348. 